\title{
Bubble nucleation and pressure generation during laser cleaning of surfaces
}

\author{
O. Yavas, A. Schilling, J. Bischof, J. Boneberg, P. Leiderer \\ Department of Physics, University of Konstanz, Postfach $5560<$ M676>, D-78434 Konstanz, Germany \\ (E-mail: Paul.Leiderer@uni-konstanz.de)
}

Received: 5 December 1996/Accepted: 6 January 1997

\begin{abstract}
Bubble nucleation and growth dynamics, on a nanosecond time scale, induced by pulsed laser heating of a liquid-solid interface are studied experimentally. A surface-plasmon probe is implemented as a novel, highly sensitive method for the study of submicroscopic bubbles, providing accurate information on the nucleation thresholds, growth velocities, and transient pressure generation by rapid bubble growth. Owing to the higher sensitivity of the surface plasmon probe to small bubbles, it is demonstrated that bubble nucleation sets in at a lower liquid superheating than previously determined with the use of optical reflectance or piezoelectric transducer measurements. A comparison of experimentally determined bubble growth velocities with computational results confirms that bubble growth is governed by the heat transfer from the solid surface into the liquid. Reconstructed surface plasmon resonance curves from transient signals are used to estimate the fractional volume and number density of bubbles in the superheated liquid layer. Further, a surface plasmon probe is utilized for the absolute measurement of bubble-growthinduced pressure amplitudes on a nanosecond time scale. The measurements yield peak pressure amplitudes in the range of $\sim 1-5 \mathrm{MPa}$ with a pressure pulse width of $\sim 40 \mathrm{~ns}$. Additionally, the phase of an acoustic pulse is observed to change upon reflection at the liquid-solid interface if bubbles are present, providing a direct proof for laser-induced bubbles.
\end{abstract}

PACS: $68.45 ; 81.60 ; 82.65 z$

The cleaning of solid surfaces, particularly the removal of submicroscopic particle contaminants, without inducing any damage to the surface, is one of the challenging tasks in microelectronic fabrication. The strong adhesion forces exerted by very small particles mean that the conventional cleaning methods, such as ultrasonics, are inefficient for the removal of submicroscopic particles, which cause significant yield losses in the manufacturing process $[1,2]$. In addition, for environmental and labor safety reasons the bulk usage of hazardous chemicals and solvents, such as CFCs (carbon fluoro chloride), in the conventional cleaning techniques becomes increasingly undesirable. Recently, laser cleaning has emerged as a potentially promising approach to overcome these drawbacks [3-8]. Not resorting to direct contact or chemical reaction, it represents a 'soft' and environment-friendly cleaning technique, capable of removing both hydrocarbon films and submicroscopic particle contaminants from solid surfaces. In 'steam laser cleaning', which is reported to give the highest efficiency for the removal of submicroscopic particles as small as $0.2 \mu \mathrm{m}$ [3-5], a thin liquid film is deposited onto the contaminated surface and is rapidly heated by pulsed laser irradiation. The subsequent explosive evaporation of the liquid film causes the removal of the contaminating particles.

The practical relevance of explosive liquid evaporation in steam laser cleaning and other technologically important areas, such as laser surgery [9], laser chemistry [10,11], and localized depassivation of metallic surfaces [12,13], has motivated research in the bubble nucleation and pressure generation processes induced by pulsed laser heating of a liquid-solid interface. Initial studies had been performed on thin liquid films by using an optical transmission probe [14], which provides useful information on temperature transients and "explosion" thresholds. However, liquid film rupture and droplet formation in the path of the probe beam make data analysis difficult and, in addition, the optical transmission probe is limited to certain semitransparent solid substrates. Therefore, in our previous studies we have applied optical reflectance, light scattering, and piezoelectric transducer probes to bulk liquids in contact with solid surfaces [15-18]. Using these test methods we have been able to clarify several aspects of bubble nucleation induced by pulsed laser heating at a liquid-solid interface. We have, for example, demonstrated that the optical properties of a foamy liquid layer can be described by Maxwell Garnett's effective-medium theory [19] when the bubbles are small compared to the probe-beam wavelength, and that Mie scattering dominates when the bubbles reach a size comparable to the probe beam wavelength, resulting in a transient decrease in the specular reflectance signal. Further, threshold fluences for bubble growth to an observable size, i.e., to the order of the probe-beam wavelength, could be determined accurately by using both optical reflectance and 
piezoelectric transducer measurements. In addition, from optical reflectance measurements the bubble growth velocities for various liquids on a $0.2-\mu \mathrm{m}$-thick chromium film could be estimated to be of the order of $\mathrm{m} / \mathrm{s}(\sim 2 \mathrm{~m} / \mathrm{s}$ for alcohols, and $\sim 4 \mathrm{~m} / \mathrm{s}$ for water). Bubble growth velocities of the same order were obtained recently via interferometric measurements [20]. Simultaneous measurements from optical temperature probes and specular reflectance have demonstrated that bubble nucleation takes place at moderate liquid superheating [21], indicating the heterogeneous nature of the nucleation process, where the solid surface provides nucleation sites for bubbles.

When the heat deposited at the liquid-solid interface diffuses away from the interface, the liquid cools down below the boiling temperature and the thermally induced bubbles collapse within a few hundred nanoseconds. On a longer, microsecond time scale, however, repeated bubble formation due to cavitation is observed. This is caused by multiple reflections of an acoustic pulse of nanosecond duration, which is generated by rapid bubble growth at the liquid-solid interface [15-18]. Using a double-pulse experiment and probing different areas of the sample surface, we have proven that an essential requirement for the observation of this 'memory effect' is the preceding thermally induced bubble formation at the liquid-solid interface $[17,18]$. It is therefore concluded that the thermally induced bubbles do not completely disappear when they collapse, but survive for a few hundred microseconds, serve as embryonic bubbles and enhance acoustic cavitation.

The limitations of the previously used test methods mean that despite their success in the study of the nucleation processes at a liquid-solid interface, as briefly described above, some problems still remain unsolved. For example, because of the lack of a bandwidth and transducer ringing, the piezoelectric transducer technique does not allow an absolute measurement of the pressure amplitudes on a nanosecond time scale, providing only qualitative results or requiring tedious calibration procedures. Furthermore, the optical-reflectance and light-scattering signals are very small until the bubbles reach a size comparable to the probe beam wavelength, and they are therefore relatively insensitive to smaller or embryonic bubbles. Here, we will present the results of our recent studies in which we used surface plasmons as a probe, which proved to be a highly sensitive tool for the study of nucleation and pressure generation processes induced by pulsed laser heating at a liquid-solid interface, providing information also on the early stages of the nucleation process and enabling the measurement of absolute pressure amplitudes on a nanosecond time scale.

\section{Experimental arrangement and procedure}

The experimental setup for the surface plasmon probe (SPP) of the laser-induced bubble nucleation at a liquid-solid interface is depicted in Fig. 1a. A thin $53 \mathrm{~nm}$ silver film is evaporated in high vacuum onto the base of a heavy flint glass prism $(n=1.7494$ for $\lambda=632.8 \mathrm{~nm})$. A 2 -nm-thick chromium interlayer is used to increase the adhesion between the silver film and the glass prism. The prism with the silver film is mounted on a cuvette filled with distilled water. Surface plasmons are excited optically at the silverwater interface via attenuated total reflection (ATR) in the
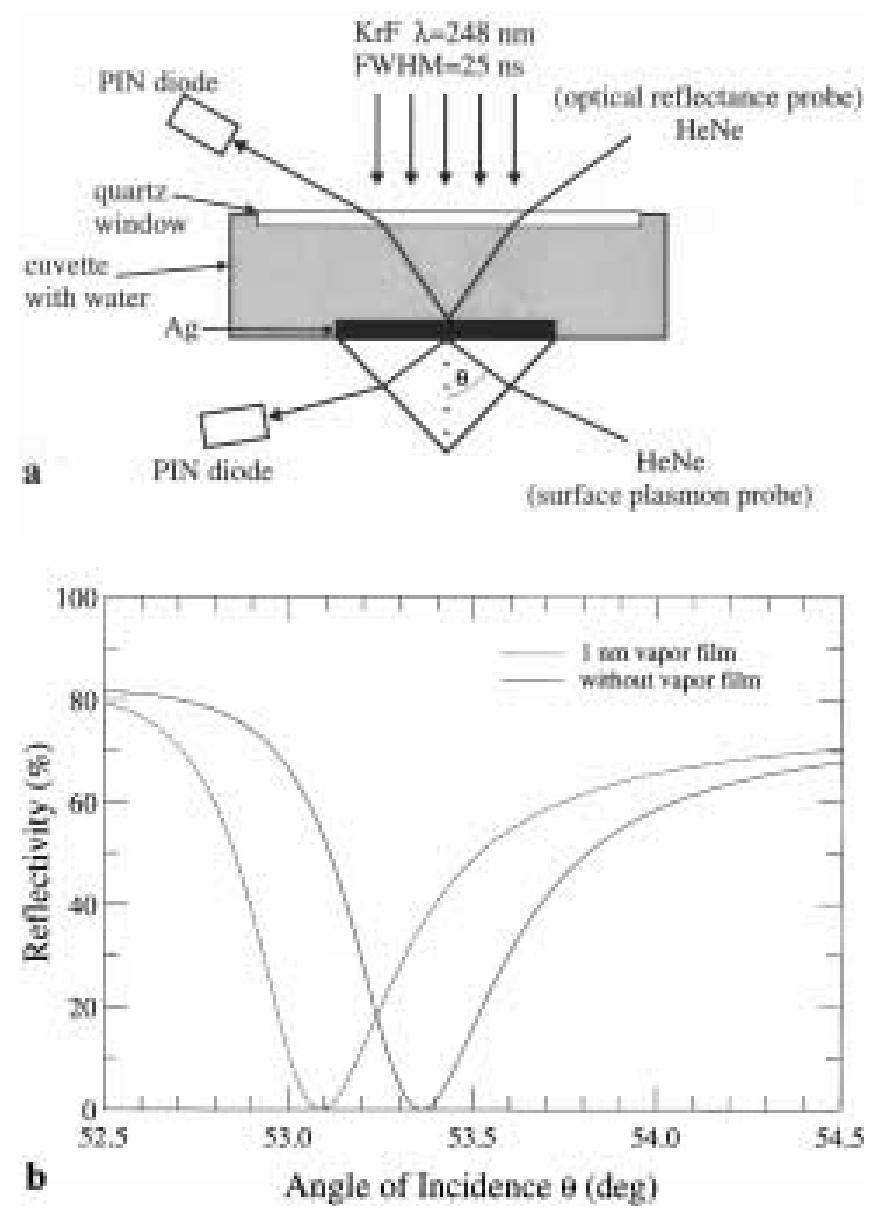

Fig. 1. a Experimental configuration for the nanosecond time-resolved study of pulsed laser-induced bubble formation at a liquid-solid interface by using a surface-plasmon probe and optical reflectance probe. $\mathbf{b}$ Calculated shift of the surface-plasmon resonance curve caused by a $1 \mathrm{~nm}$ thick vapor film at a water-silver interface

Kretschmann configuration via a $5 \mathrm{~mW}$ HeNe probe laser [22]. Bubble nucleation takes place at the silver-water interface upon heating by a $\mathrm{KrF}$ excimer laser pulse $(\lambda=248 \mathrm{~nm}$, FWHM $\approx 25 \mathrm{~ns}$, spot size $1 \times 1 \mathrm{~cm}^{2}$ ) through a quartz window. Any transient change in the surface-plasmon resonance angle due to a change in the dielectric function of the water layer on the silver film, induced by bubble nucleation, a temperature rise, or pressure changes, is monitored using a fast photodiode (rise time $\leq 1 \mathrm{~ns}$ ). The signal is amplified by using an ac-coupled 1-GHz-bandwidth amplifier, and recorded on a $500 \mathrm{MHz}$ digitizing storage oscilloscope. As indicated in Fig. $1 \mathrm{~b}$ it is calculated that a vapor layer of $1 \mathrm{~nm}$ thickness will cause a significant shift in the surface-plasmon resonance to a smaller angle of incidence. In order to directly compare the results of the novel SPP with the previously used optical reflectance probe (ORP), a second HeNe probe laser, incident from the front side through the liquid and probing the same spot as the SPP, is used to monitor simultaneously the transient reflectance changes caused by light scattering from bubbles. If not stated otherwise, the SPP measurements presented in the following have been performed by setting the angle of incidence for the probe beam to the middle of the left wing of the surface plasmon resonance, which is located at about $53^{\circ}$ as shown in Fig. 1b. In order to verify that no reversible changes in the optical constants of the 
silver film take place upon laser heating, the plasmon resonance curve is measured prior to and after each series of experiments.

\section{Experimental results and discussion}

\subsection{Nucleation threshold}

As already mentioned, several effects may influence the position and shape of the surface-plasmon resonance. In order to determine the contribution of the temperature rise in the silver film, experiments have been conducted first with the bare silver film in the absence of water. The result for a laser irradiation with a fluence of $F=20 \mathrm{~mJ} / \mathrm{cm}^{2}$ is shown in Fig. 2 (top curve). Owing to the combined effects of plasmon-resonance shift and broadening, the temporal shape of the probe signal depends on the angle of incidence for the probe beam. A thorough discussion of this behavior is given by Herminghaus and Leiderer [23]. The observed reflectance changes are relatively small, being about $3 \%$ even for the highest laser fluence used, $F=32 \mathrm{~mJ} / \mathrm{cm}^{2}$, for which numerical computations result in a peak temperature of $T=746 \mathrm{~K}$ in the silver film. The temporal shape of the reflectance signal is governed by the sudden temperature rise in the silver film as a result of rapid laser heating in a few nanoseconds and the subsequent gradual decrease in the temperature because of heat transfer on a microsecond time scale.

The amplitude of the reflectance drop is drastically increased when the experiment is conducted with the cuvette filled with water. As calculations show, the change in the optical properties of the dielectric half space (in our case water) as the result of a given temperature rise [25] causes a much larger shift in the surface-plasmon resonance than a change in the optical properties of the silver film induced by the same temperature rise [26]. Consequently, the resulting shape of the SPP is predominantly governed by the changes in the refractive index of water with temperature. A comparison of the transient SPP signals acquired in the absence and in the presence of water is given in Fig. 2. Note that in the presence of water the

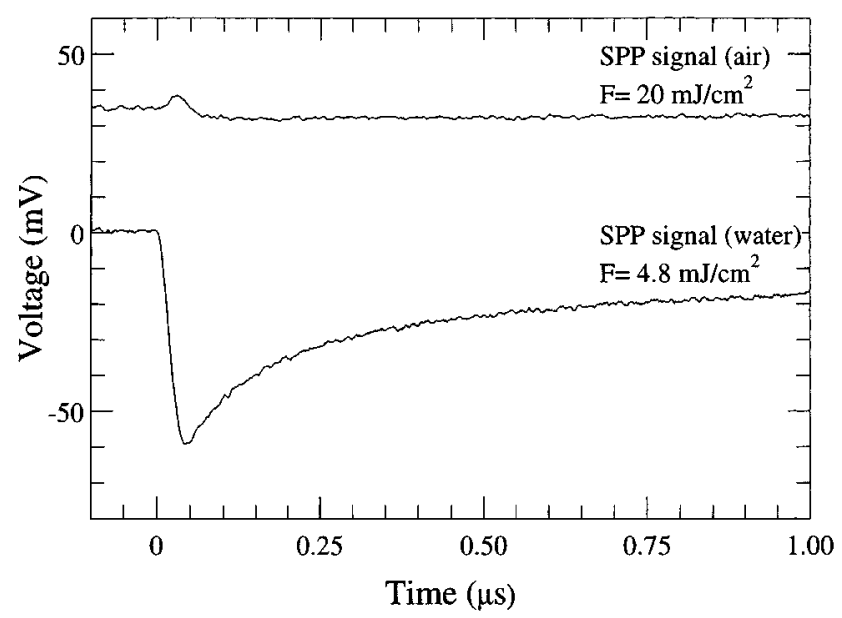

Fig. 2. Comparison of surface-plasmon probe signals acquired in the absence (topcurve) and in the presence (bottomcurve) of water in the cuvette. $10 \mathrm{mV}$ corresponds to a reflectance change of $3 \%$. The curves are offset for clarity observed reflectance change amounts to $18 \%$, which, despite the five-times-lower laser fluence used, is six times higher than that in the case of laser irradiation of the bare silver surface. As expected, a transient decrease in the SPP signal is observed because of a shift in the surface-plasmon resonance to smaller angles with increasing temperature. As long as the laser fluence is below a certain threshold (which corresponds to the threshold for bubble nucleation, see below), the SPP signal exhibits no particular extra features.

When the excimer laser fluence is increased further, however, an additional structure in the SPP signal is observed as shown in Fig. 3a. A hump in the reflectance drop starts to appear at an excimer laser fluence of $F=10.5 \mathrm{~mJ} / \mathrm{cm}^{2}$, and is more pronounced with increasing laser fluence. The appearance of this hump can be interpreted as the onset of bubble nucleation at the water-silver interface. The surface-plasmons are effectively scattered by the bubbles and, consequently, the surface-plasmon resonance is broadened and shifted, resulting in a temporary increase in the reflectance. When the bubbles collapse, the surface-plasmon resonance becomes narrower and the reflectance decreases to a level given by the resonance shift due to the actual temperature present at the water-silver interface. For the laser fluence of $F=10.5 \mathrm{~mJ} / \mathrm{cm}^{2}$, numerical computations yield a peak temperature of $T=384 \mathrm{~K}$ at the water-silver interface, i.e., a surprisingly low liquid su-
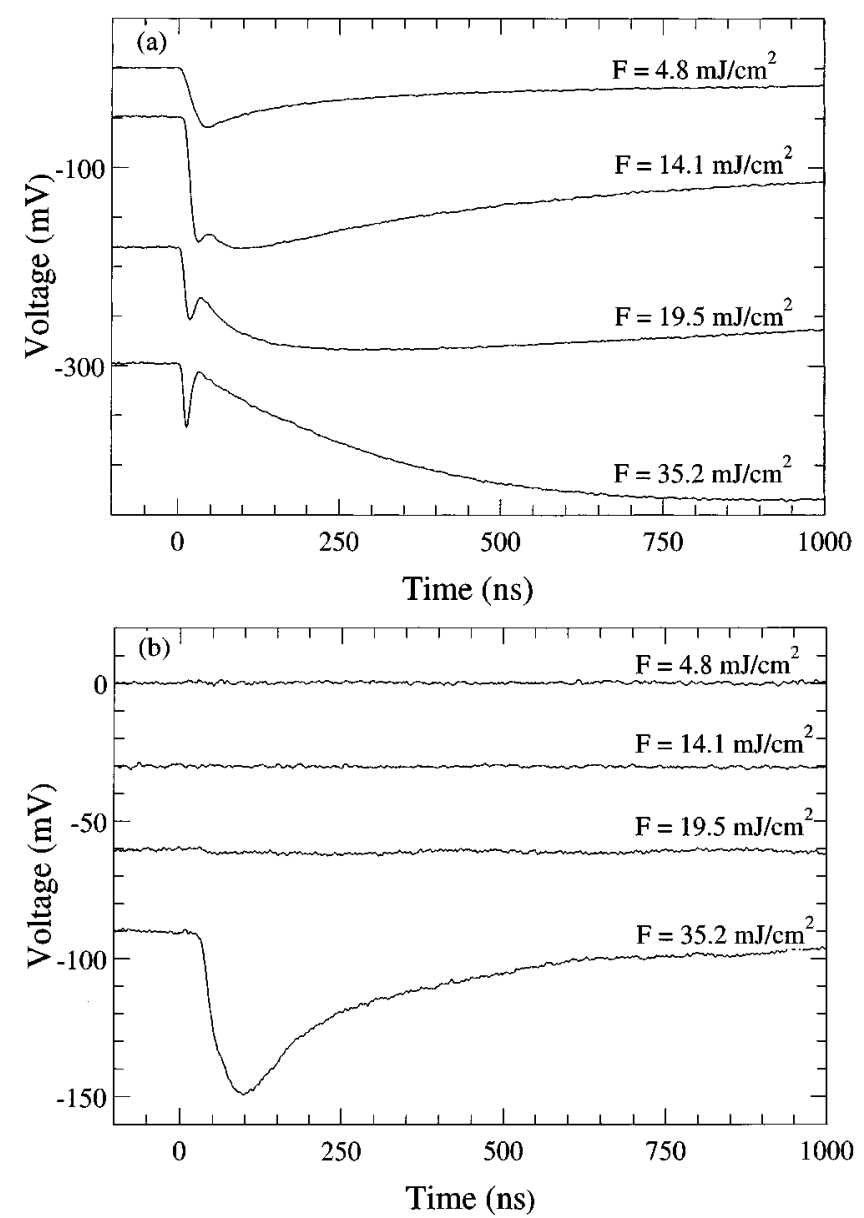

Fig. 3. a Surface-plasmon probe, and (b) optical reflectance signals for the water-silver interface with increasing excimer laser fluence. $10 \mathrm{mV}$ corresponds to a reflectance change of $3 \%$. The curves are offset for clarity 
perheat of about $\sim 10 \mathrm{~K}$. This superheat is of the same order as recently concluded for a similar system [21]. Although the hump in the SPP signal starts to appear at $F=10.5 \mathrm{~mJ} / \mathrm{cm}^{2}$ and becomes more pronounced with increasing laser fluence, the simultaneously acquired ORP signals exhibit no particular features up to the fluence level of $F=19.5 \mathrm{~mJ} / \mathrm{cm}^{2}$. Only at this threshold fluence, for which computations predict a peak temperature of $T=473 \mathrm{~K}$ at the water-silver interface (liquid superheating $\sim 100 \mathrm{~K}$ ), does a transient decrease in the ORP signal start to appear as shown in Fig. 3b. This result strikingly demonstrates the high sensitivity of the surface-plasmon probe to small bubbles that collapse before they grow to a size of the order of the probe-beam wavelength and therefore remain invisible to the ORP. It has been previously pointed out that the thickness of the superheated liquid layer is a limiting factor for the maximum bubble size achievable [27]. The difference in the threshold fluences acquired by using these two different methods can be explained by assuming that the threshold temperature obtained from the SPP corresponds to the temperature where the bubble nucleation sets in, and the threshold temperature obtained from the ORP corresponds to the temperature where the bubble growth becomes effective.

The interpretation presented above is supported by the results of numerical computations, as depicted in Fig. 4. The calculated temperature profile in water is shown for two different $\mathrm{KrF}$ excimer laser fluences. A detailed description of the computations, in which any phase change, such as bubble formation, has been neglected, can be found elsewhere $[18,24]$. It has been recently verified that the results of the computations agree well with the experimentally obtained temperature profiles at the interface [21]. Here, for $F=10.5 \mathrm{~mJ} / \mathrm{cm}^{2}$, the threshold fluence obtained with SPP, the thickness of the superheated water layer adjacent to the silver surface is calculated to be $\sim 20 \mathrm{~nm}$, i.e., the largest bubble size achievable is limited to $\sim 20 \mathrm{~nm}$ if we assume that in a first approximation the bubbles contained in the superheated liquid layer are of spherical form. This result demonstrates the ability

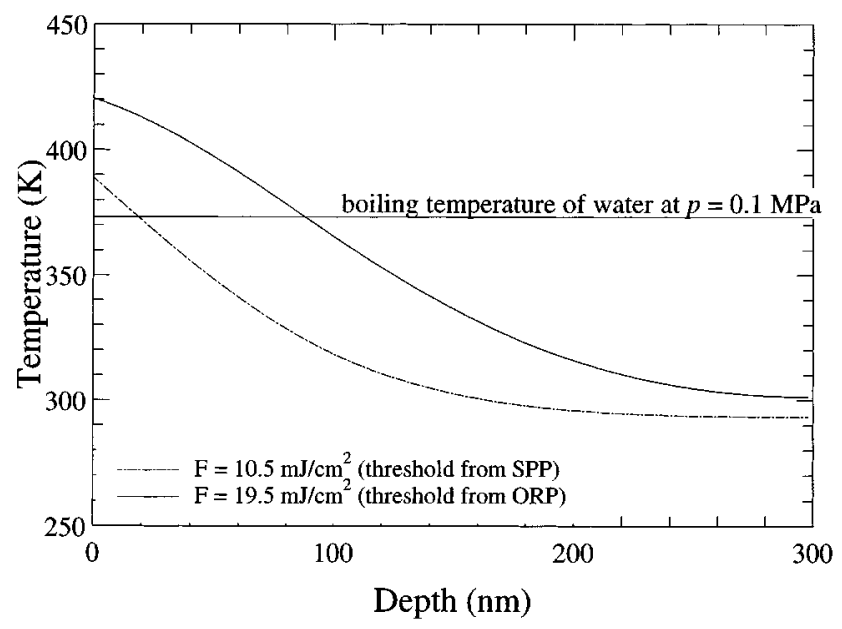

Fig. 4. Computed temperature profiles in water for two different excimer laser fluences. For $F=10.5 \mathrm{~mJ} / \mathrm{cm}^{2}$, the threshold fluence obtained via SPP, the thickness of the superheated water layer amounts to $\sim 20 \mathrm{~nm}$ at $44 \mathrm{~ns}$ after the onset of the laser irradiation. For $F=19.5 \mathrm{~mJ} / \mathrm{cm}^{2}$, the threshold fluence obtained with ORP, the thickness of the superheated water layer amounts to $\sim 90 \mathrm{~nm}$ at $75 \mathrm{~ns}$ after the onset of the laser irradiation of SPP to detect bubbles as small as $\sim 20 \mathrm{~nm}$ in diameter with nanosecond time resolution, whereas these bubbles remain invisible to ORP within the resolution of the experiments $\left(\Delta R / R \sim 10^{-3}\right)$. This is somewhat surprising since it has been reported that the SPP is in principle able to detect even submonolayers of adsorbed dielectric films [28, 29]. In contrast to the expected huge changes in the SPP signal upon bubble nucleation and growth (see Fig. 1b), the observed changes are relatively small. This is attributed to a low surface coverage of bubbles, i.e., to the low bubble density at the surface, as will be discussed in Sect. 2.3. For $F=19.5 \mathrm{~mJ} / \mathrm{cm}^{2}$, the threshold fluence obtained from ORP, the thickness of the superheated water layer is calculated to be $\sim 90 \mathrm{~nm}$, i.e., in this case the largest bubble size achievable is $90 \mathrm{~nm}$. Hence, ORP first starts to detect bubbles when they reach a size of $\sim 90 \mathrm{~nm}$. It is known that at a bubble radius, $R$, comparable to this value $(R \sim 80 \mathrm{~nm}$, for $\lambda=632.8 \mathrm{~nm})$ Mie scattering becomes effective [30,31], resulting in a transient decrease in the reflectance signal [15-18]. The good agreement between the theoretically predicted bubble-radius threshold at which Mie scattering becomes effective and the thickness of the superheated liquid layer for the threshold laser fluence leads us to the conclusion that bubble growth is governed by the heat transfer from the solid surface into the liquid. The bubbles remain confined within the superheated liquid layer and the bubble size does not exceed the superheated liquid layer thickness.

\subsection{Bubble growth velocity}

As mentioned above, in our previous studies transient ORP signals were used to estimate bubble growth velocities, which were found to be of the order of $\mathrm{m} / \mathrm{s}$. These growth velocities were confirmed independently via interferometric measurements by Kim et al. [20]. In the last section it has been shown that bubble growth is governed by the heat transfer from the solid surface into the liquid. Accordingly, except in the very early, inertia-controlled stage of bubble growth [32], the growth velocity will be limited by the speed of heat transfer from the solid surface into the liquid. Based on this fact, the bubble growth velocity can be extracted from the results of

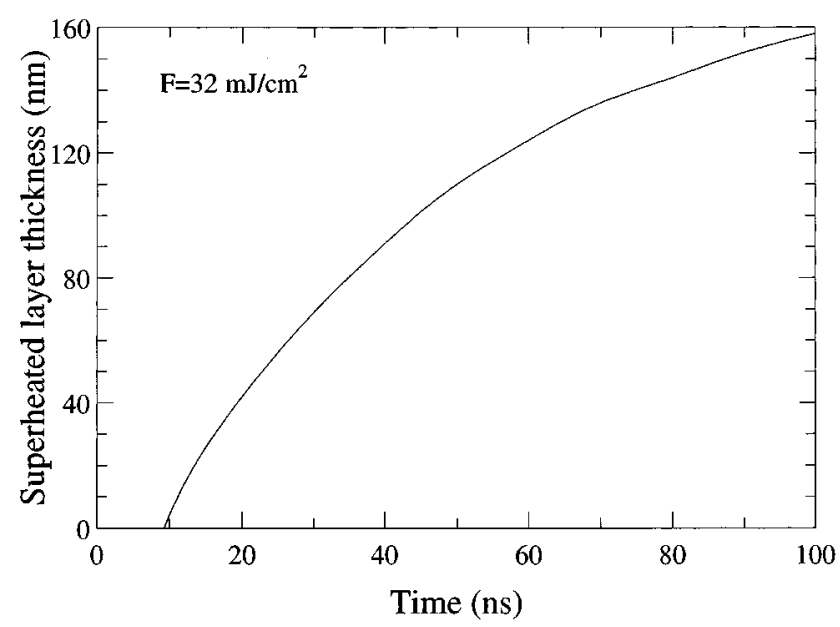

Fig. 5. Computed thickness of the superheated water layer as a function of time for the excimer laser fluence of $F=32 \mathrm{~mJ} / \mathrm{cm}^{2}$ 
the numerical computations $[18,24]$. The computed evolution of the superheated liquid layer thickness as a function of time for a laser fluence of $F=32 \mathrm{~mJ} / \mathrm{cm}^{2}$ is depicted in Fig. 5. As expected for a diffusion process, the speed at which the thickness of the superheated liquid layer increases exhibits a $\sqrt{t}$ dependence. However, for the initial $50 \mathrm{~ns}$ following the onset of the laser irradiation an average growth velocity of about $2.6 \mathrm{~m} / \mathrm{s}$ is obtained, which agrees quite well with the experimentally determined bubble growth velocities for a similar system, i.e., water on chromium, in previous studies [15-18].

\subsection{Fractional volume and number density of bubbles}

Any change in the real part of the dielectric function at the water-silver interface results in a shift in the surface-plasmon resonance curve, and scattering that is induced by the bubbles at the interface can also cause a broadening of the resonance. An important implication of the results presented in Sect. 2.1 is that in the fluence range used any film boiling can be excluded, since otherwise above the nucleation threshold the SPP signal would exhibit a different behavior than observed. As already mentioned, any film boiling would result in a substantial shift in the surface-plasmon resonance curve to a smaller angle of incidence, as indicated in Fig. $1 \mathrm{~b}$ for a 1-nm-thick vapor film. However, a shift of this extent has not been observed. Therefore, it can be assumed that in our case the nucleation process is heterogeneous, and the formation of separate bubbles at individual nucleation centers on the silver surface is expected, rather than a homogeneous vapor-film formation. Consequently, it is expected that a transient broadening of the surface-plasmon resonance curve will be superimposed to a shift in the resonance curve as bubbles are nucleated at the water-silver interface. Unfortunately one single SPP measurement at a fixed angle of incidence does not provide sufficient information to separate these two effects.

In order to study the time dependence of the surface plasmon resonance characteristics during the bubble nucleation process, one needs a sequence of resonance curves, as in Fig. 1b, on a nanosecond time scale. Since it is difficult to scan the angle of incidence so rapidly, we have acquired a series of transient SPP signals at different fixed angles, and have reconstructed the surface-plasmon resonance curve for various instants following the onset of the excimer laser pulse. In this way we could obtain 'snapshots' of the surface-plasmon resonance curve during bubble growth and collapse. In order to assure reproducible nucleation dynamics, care was taken to apply exactly the same excimer laser fluence at each angle of incidence for the SPP beam. The resonance curves are then reconstructed by determining the change in the SPP signal from its initial level for all the acquired signals at the considered time instant. The resulting surface-plasmon resonance curves for various time instants are shown in Fig. 6. For this series of experiments the excimer laser fluence has been kept constant at $F=32 \mathrm{~mJ} / \mathrm{cm}^{2}$. In the first few hundred nanoseconds following the excimer laser pulse the resonance curve exhibits a slight shift to smaller angles of incidence and simultaneously a substantial broadening. This result is consistent with the formation of bubbles at the water-silver interface following laser irradiation as discussed above. The bubbles represent effective scattering centers for the surfaceplasmons and cause a resonance broadening. When after a
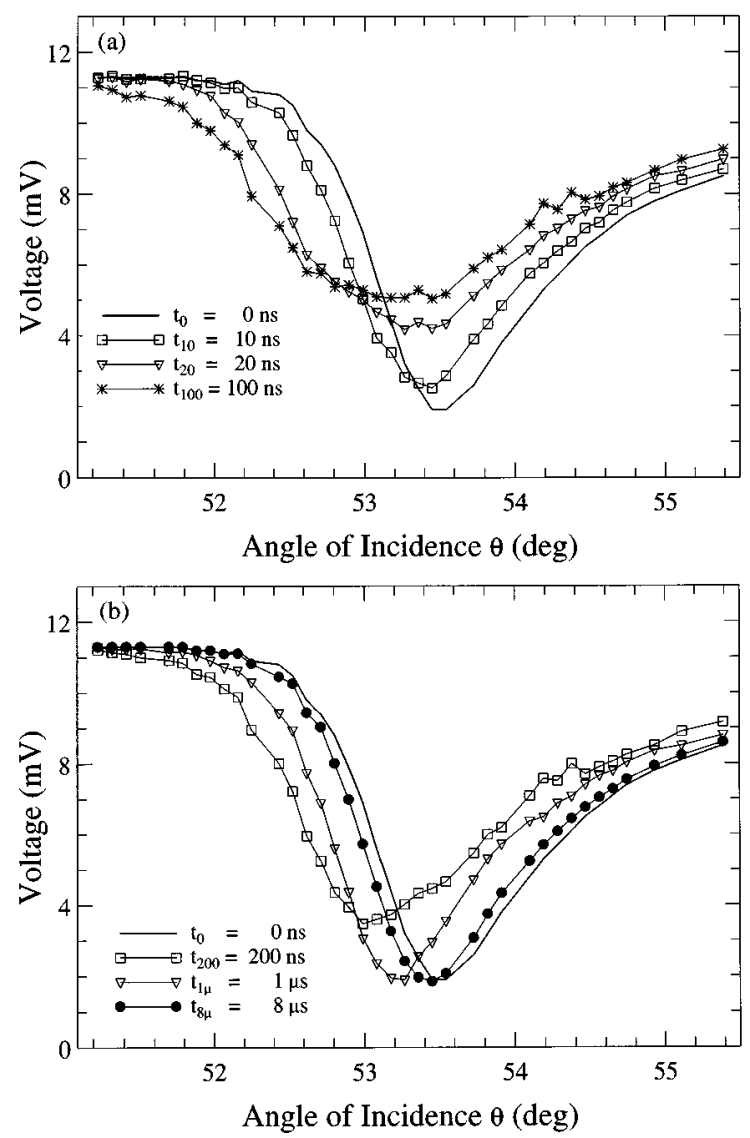

Fig. 6a,b. Reconstructed surface-plasmon resonance curves at various time instants (snap-shots): a short-time-scale measurements, and (b) long-timescale measurements. $F=32 \mathrm{~mJ} / \mathrm{cm}^{2}$

few hundred nanoseconds the bubbles collapse and disappear the initial width of the resonance curve is restored. Consequently, on the microsecond time scale the resonance curve is shifted to smaller angles of incidence only because of the temperature rise in the water, which decays in several tens of microseconds to its initial level as heat diffuses out from the interface.

The interpretation that after the collapse of bubbles the surface-plasmon resonance shift is caused solely by the temperature rise is supported also by the results of numerical computations for the temperature evolution at the interface. The computation for the excimer laser irradiation with $F=32 \mathrm{~mJ} / \mathrm{cm}^{2}$ yields a temperature rise of $18 \mathrm{~K}$ within the probe depth after $8 \mu$ s following the onset of the laser pulse. For this temperature rise in water we have calculated the expected shift of the surface-plasmon resonance curve by using the transfer matrix method for a multilayer system [31]. The optical constants for water as a function of temperature are taken from the literature [24]. The calculated resonance shift of $\Delta \Theta=0.12^{\circ}$ compares well with the experimentally determined $\Delta \Theta=0.08^{\circ}$. Accordingly, the temporal shape of the SPP signal in Fig. 3a can be well described: initially, the laser irradiation causes a rapid temperature rise in the water, resulting in a shift in the resonance curve and a decrease in the SPP signal. When bubbles are formed at the interface, the resonance curve is broadened, leading to a temporary increase in the SPP signal (hump). Afterwards, when the bubbles have col- 
lapsed, the SPP signal is determined only by the temperature profile present, and returns to its initial level as heat diffuses from the interface.

The reconstructed surface-plasmon resonance curves could be used to roughly estimate the fractional volume of bubbles in the superheated water layer. For this purpose, first, the shift in the resonance curve has been determined experimentally for the time instant $10 \mathrm{~ns}$ after the excimer laser pulse. Taking into account the shift in the resonance curve due to the actual temperature profile present at the interface (obtained from the heat-flow calculations), we determine the shift in the resonance curve due to bubble formation to be $\Delta \Theta \approx 0.05^{\circ}-0.1^{\circ}$ at most. In our previous studies [15-18] we have demonstrated that the foamy layer can be described by Maxwell Garnett's effective-medium theory [19], according to which the effective dielectric constant of the foamy layer, $\varepsilon_{\text {eff }}$, depends on the fractional volume of bubbles, $f$, as

$\varepsilon_{\mathrm{eff}}=\varepsilon_{1}\left(1-\frac{3 f\left(\varepsilon_{1}-\varepsilon_{\mathrm{v}}\right)}{2 \varepsilon_{1}+\varepsilon_{\mathrm{v}}+f\left(\varepsilon_{1}-\varepsilon_{\mathrm{v}}\right)}\right)$.

The subscripts 1 and $\mathrm{v}$ refer to liquid and vapor, respectively. In order to determine the fractional volume of bubbles giving rise to the experimentally obtained shift in the surfaceplasmon resonance curve, the effective dielectric constant for a 5-10-nm-thick foamy layer has been calculated as a function of $f$ and the resulting shift of the resonance curve has been computed by using the transfer matrix method. For $f=0.05$ the computations yield resonance shifts of $\Delta \Theta=0.045^{\circ}$ and $\Delta \Theta=0.089^{\circ}$ for a 5 - and a 10 -nm-thick foamy layer, respectively. For $f=0.1$ the resonance shift for a 5-nm-thick foamy layer is calculated to be $\Delta \Theta=0.093^{\circ}$. Since these calculated shifts compare well with the experimentally determined shifts in the surface-plasmon resonance curve, it can be concluded that at $10 \mathrm{~ns}$ following the onset of the excimer laser pulse the upper limit for the fractional volume of bubbles in the superheated water layer amounts to $f \approx 0.05-0.1$.

In the preceding sections it has been demonstrated that bubble nucleation takes place at the solid surface and the bubble growth is governed by heat diffusion into the liquid. Accordingly, the maximum bubble size corresponds approximately to the thickness of the superheated liquid layer. Under this assumption the number density of bubbles, $N$, can be easily estimated. For the estimated fractional volume of $f \approx 0.01-0.05$, the number density of bubbles in the irradiated area is calculated to be $N \sim 10^{9} \mathrm{~cm}^{-2}$.

\subsection{Pressure generation due to rapid bubble growth}

In our previous studies in which we used piezoelectric transducers we have demonstrated the generation of high-intensity acoustic pulses on a nanosecond time scale as a result of rapid bubble growth [16-18]. This enhancement of the acoustic pulse intensity due to the rapid bubble-growth process is thought to play a major role in the removal of submicroscopic particles during laser cleaning. Since the piezoelectric transducer measurements could not provide the absolute pressure amplitudes, a quantitative data analysis is not yet possible.

However, we have recently succeeded in applying the surface-plasmon probe to absolute pressure measurements on a nanosecond time scale $[33,34]$. In order to eliminate any (a)

$\mathrm{KrF} \lambda=248 \mathrm{~nm}$ FWHM $=25 \mathrm{~ns}$
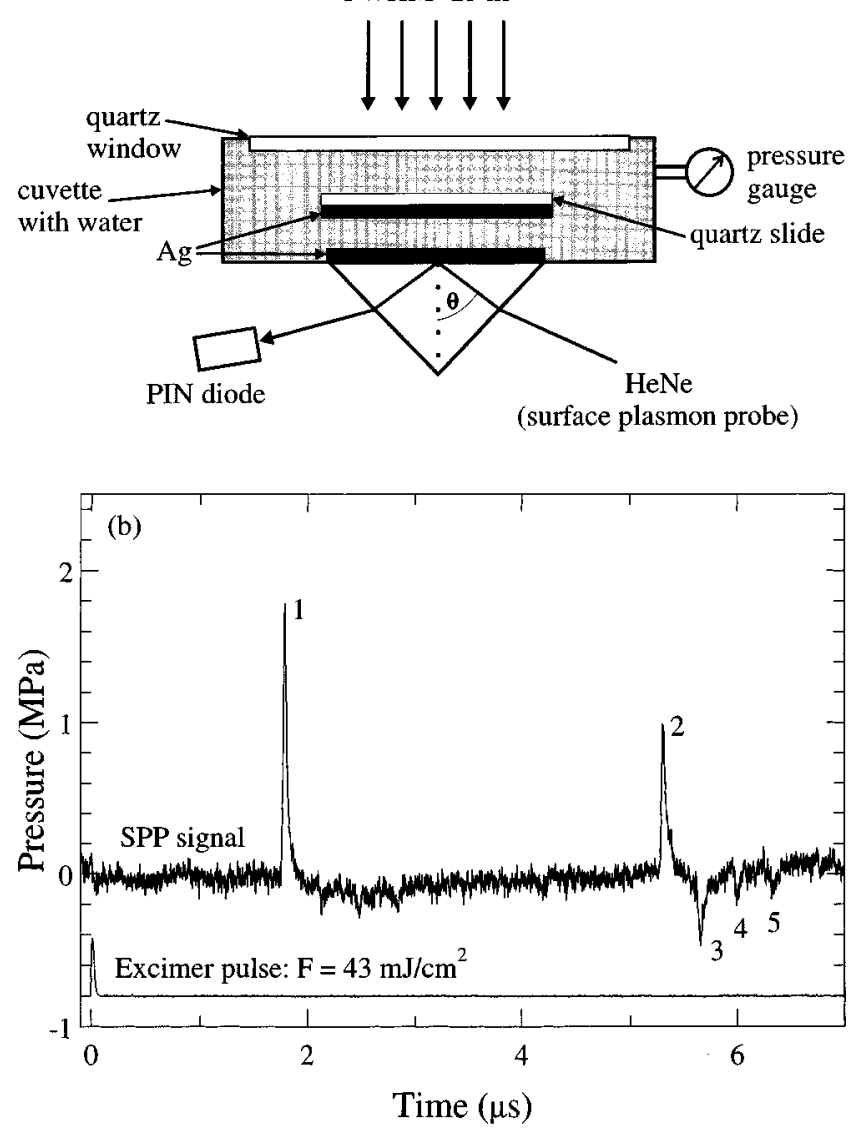

Fig. 7. a Experimental configuration for the absolute measurement of acoustic pulse amplitudes on a nanosecond time scale by using a surface-plasmon probe. In order to eliminate thermal effects on the silver film, the pressure pulse is generated on the quartz slide by excimer laser irradiation. b Transient pressure signal acquired using the surface-plasmon probe

temperature effects, the experimental setup presented in Fig. 1 has been slightly modified as shown in Fig. 7a. A thin quartz slide is placed in the water at a variable distance from the prism. The quartz slide is coated with $20 \mathrm{~nm}$ of chromium and $60 \mathrm{~nm}$ of silver on the surface facing the prism. The irradiation of the quartz slide from the rear side results in the sudden heating of the silver film and in bubble nucleation at the silver-water interface. The acoustic pulse generated during this process propagates toward the prism and can be detected by the SPP. The known relation for the dielectric constant for water as a function of pressure [25] is utilized to extract the absolute pressure amplitudes from the shift in the surface-plasmon resonance curve. An example for the transient pressure signal acquired by using this setup is presented in Fig. 7b. A detailed description of the data acquisition system and the analysis of the SPP signals, i.e., conversion into absolute pressure amplitudes, is given elsewhere [33, 34]. The signal exhibits several peaks according to the propagation of the acoustic pulse in the water and its repeated reflection between the prism and the quartz slide. The first peak corresponds to the acoustic pulse generated at the silver-water interface after its propagation to the SP detector film. The second peak represents the acoustic pulse after its round-trip between the prism and the quartz slide, reflected at the water- 
silver interface. Part of the acoustic pulse is transmitted into the quartz slide and is reflected back at the quartz-water interface; it is detected as the third peak in the SPP signal. Since quartz is acoustically more dense than water, the reflection at the quartz-water interface leads to a phase change and the compressive wave is converted into a tensile one, manifested in a negative pressure signal. The successive peaks 4 and 5 are caused by multiple reflections of the acoustic pulse in the quartz slide. By changing the distance between quartz slide and the prism, and by using quartz slides of different thicknesses, we have verified that the peaks in the SPP signal represent multiple reflections of the acoustic pulse as described.

The width of the acoustic pulse is measured to be about $40 \mathrm{~ns}$, which is much shorter than the pulse width of $\sim 100 \mathrm{~ns}$, previously measured by using a photoacoustic probe beam deflection technique [35]. This demonstrates the higher time resolution of the SPP. The localized probe depth $(\sim 200 \mathrm{~nm})$ means the SPP provides the real pulse profiles, whereas the photoacoustic deflection probe generally detects a larger pressure pulse width because of its integrating nature, i.e., the finite probe beam waist and the interaction length of the probe beam [35]. Further, the peak amplitude of the ini-
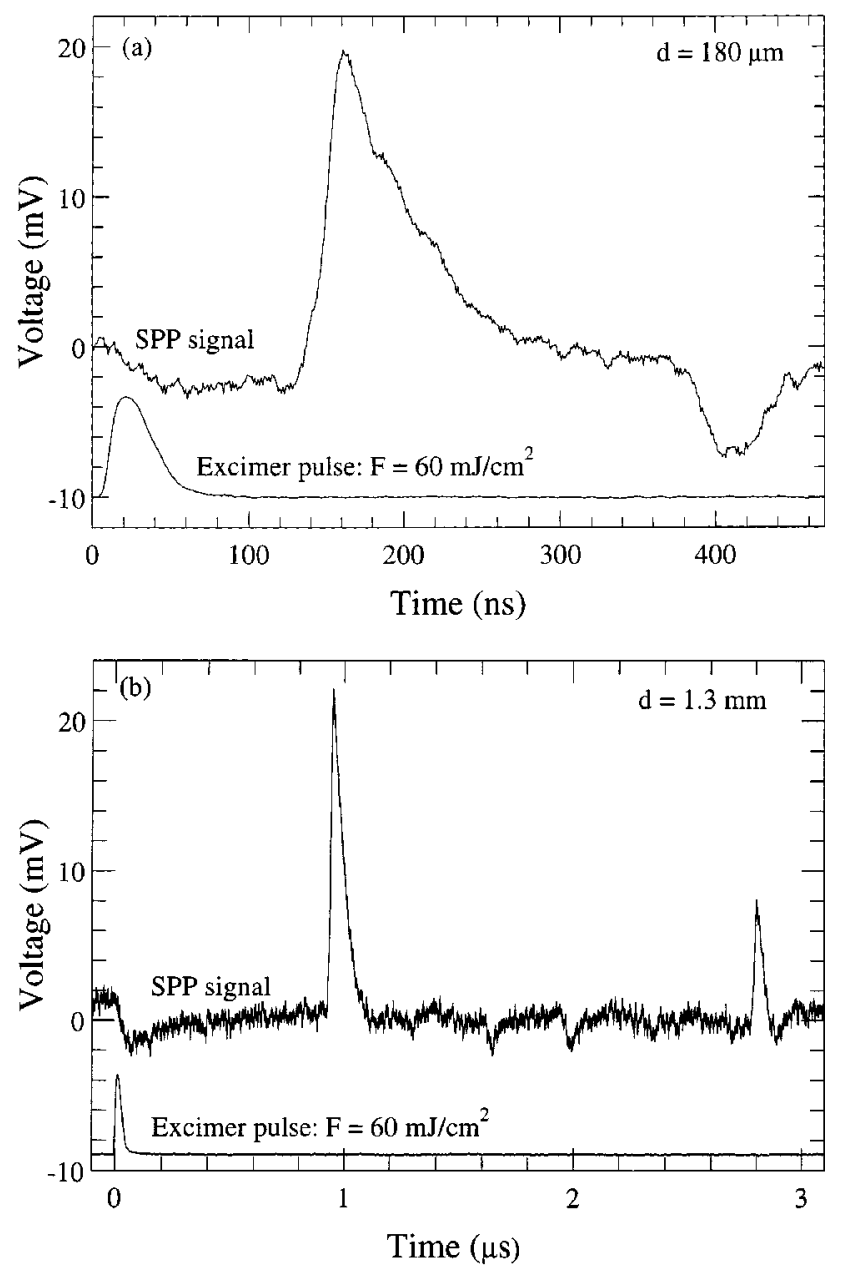

Fig. 8. a Direct proof of bubbles by the phase reversal of the acoustic pulse upon reflection at the water-silver interface when bubbles are present. b At a longer time scale, when bubbles are collapsed, reflection takes place without phase reversal tial pulse amounts to $\sim 2 \mathrm{MPa}$ for the laser irradiation with $F=43 \mathrm{~mJ} / \mathrm{cm}^{2}$, and in the successive echoes its intensity gradually decreases because of reflection and absorption losses. A pressure amplitude of the same order, but about two to three times lower, has been previously measured for a similar system in which photoacoustic probe beam deflection and piezoelectric transducer techniques where used [35]. The small discrepancy in the measured pressure amplitudes can be attributed to the different samples used and to the difference in the temporal resolution of the test methods.

\subsection{Direct proof for laser-induced bubbles}

The fact that the reflection of the acoustic pulse at an acoustically less dense medium leads to a phase change, i.e., to the conversion of the compressive pulse into a tensile one, could be used to directly prove the generation of bubbles by the excimer laser heating of the water-silver interface. As long as bubbles are present at the water-silver interface on the quartz slide, the acoustic pulse will be reflected as a tensile wave, since the reflection will take place at a watervapor interface. When the bubbles collapse and the reflection takes place at the water-silver interface, the reflected pulse will be compressive. This has been verified by placing the quartz slide near to the prism and successively increasing the distance after each excimer laser pulse. Representative results are depicted in Fig. 8 and clearly demonstrate the existence of bubbles up to $\sim 900 \mathrm{~ns}$ following the excimer laser pulse, manifested by a tensile reflected acoustic pulse up to this time instant and by a compressive reflected pulse afterwards. Although our previous studies already delivered the clear evidence for laser-induced bubbles, the results presented here provide a more direct proof for laser-induced bubbles at a liquid-solid interface. The amplitude of the reflected pressure pulse as a function of the distance between the quartz slide and the sample surface (first echo) is plotted in Fig. 9. The same excimer laser fluence was applied at each placing. Obviously the pressure amplitude starts to saturate at a distance of $1.3 \mathrm{~mm}$. This corresponds to the complete collapse of

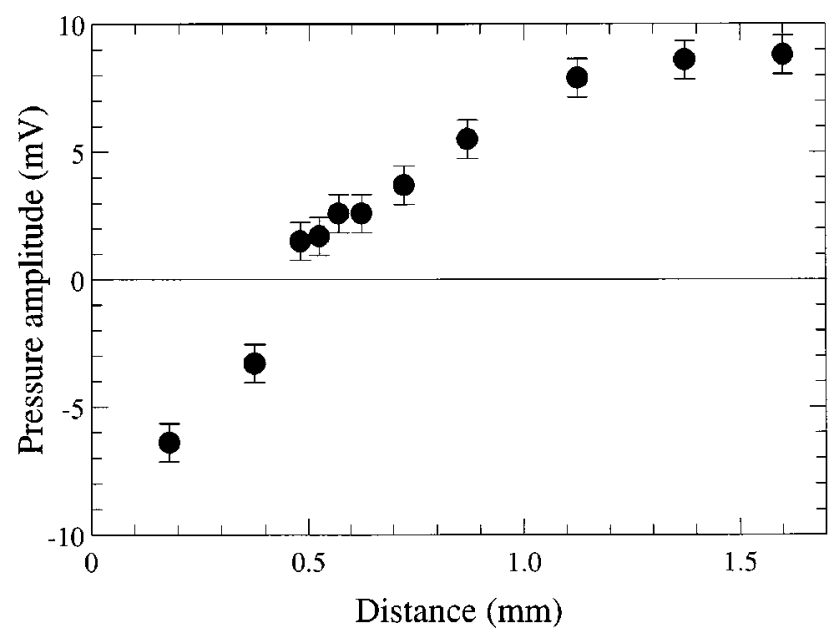

Fig. 9. The amplitude of the reflected pressure pulse as a function of the distance of the quartz slide from the SPP sample surface. Phase reversal occurs as long as bubbles are present on the surface up to $\sim 900 \mathrm{~ns}$ following the excimer laser pulse 
the bubbles after about $900 \mathrm{~ns}$. At a distance of $0.2 \mathrm{~mm}$, the smallest distance that could be achieved in this experimental setup, i.e., after about $150 \mathrm{~ns}$, the peak amplitude of the reflected tensile pulse already decreases.

Piezoelectric transducer and photoacoustic probe beam deflection techniques have previously demonstrated that explosive bubble growth results in an enhancement of the acoustic pulse intensity [16-18, 20,35]. At low laser fluences when no bubble nucleation takes place a low intensity acoustic pulse is generated by the thermoelastic expansion, whereas at higher laser fluences when explosive bubble growth takes place the intensity of the acoustic pulse is considerably increased. Similarly, the SPP technique could also be used to monitor these two different pressure-generation processes, the results exhibiting the same behavior as can be seen in Fig. 10. The higher the laser fluence, the larger and faster the bubbles should grow (in principle, the higher liquid superheating also means more nucleation sites could be activated, i.e., more bubbles could be nucleated), resulting in a higher pressure amplitude. The onset of a plateau, however, indicates that in the fluence range used a saturation sets in for the maximum achievable size, growth velocity, and/or number density of bubbles.

\section{Conclusions}

The mechanisms of bubble nucleation and growth at a liquidsolid interface have been studied quantitatively with a time resolution of nanoseconds. A surface-plasmon probe has been successfully implemented as a novel, highly sensitive method for the study of submicroscopic bubbles. Comparative studies with an optical reflectance probe and numerical computation of the laser-induced heat profiles at the liquid-solid interface have allowed us to establish a bubble nucleation and growth model, that suggests that heterogeneous nucleation takes place at the solid surface and that the bubble growth velocity is limited by speed of the heat diffusion into the liquid. The ability of the surface-plasmon probe to monitor

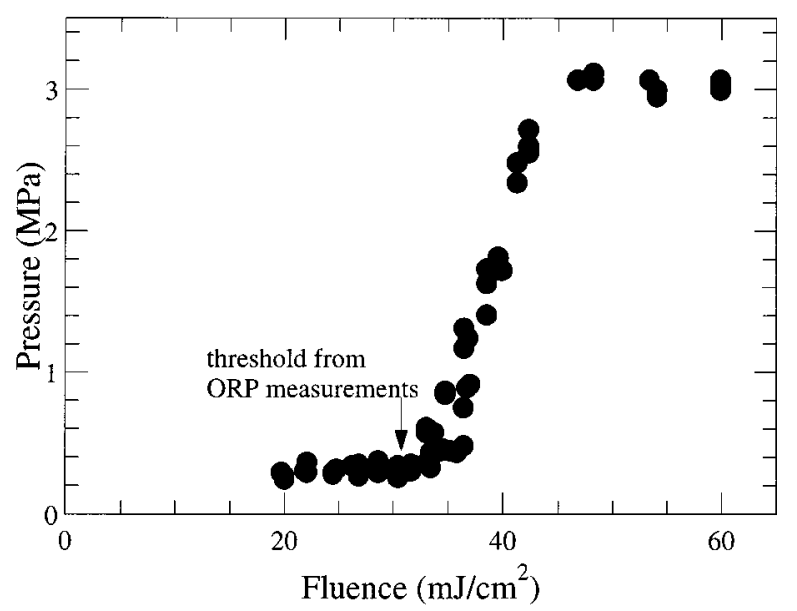

Fig. 10. Peak pressure amplitude as a function of the excimer laser fluence, demonstrating enhanced pressure generation by rapid bubble growth. The data points are measured using the surface-plasmon probe and the arrow marks the threshold for bubble growth as determined by using the optical reflectance probe bubbles as small as $\sim 20 \mathrm{~nm}$ in diameter has been demonstrated, whereas the optical reflectance probe first detects bubbles first when they reach a size of $\sim 90 \mathrm{~nm}$. Owing to the higher sensitivity of the surface-plasmon probe to small bubbles, it could be demonstrated that bubble nucleation sets in at a lower liquid superheating than previously determined with optical reflectance or piezoelectric transducer measurements. Further, reconstructed surface-plasmon resonance curves from transient signals were used to estimate the fractional volume and number density of the bubbles in the superheated liquid layer. Moreover, for the first time, the surface-plasmon probe has been successfully applied for a quantitative measurement of the pressure pulses generated by rapid bubble growth, yielding a pressure pulse width of $\sim 40 \mathrm{~ns}$ and a peak pressure amplitude in the range of $\sim 1-5 \mathrm{MPa}$.

Acknowledgements. Support of this work by the Center of Modern Optics at the University of Konstanz, Germany, is gratefully acknowledged. The authors thank S. Herminghaus at the Max-Planck Institute for Colloid and Interface Research, Berlin, Germany, Hee K. Park at the Vanderbilt University, Tennessee, and C.P. Grigoropoulos at the University of California at Berkeley, California, for fruitful discussions and useful suggestions.

\section{References}

1. S.A. Hoenig: In Particles on Surfaces, ed. K.L. Mittal (Plenum, New York, 1988), Vol.1, p.3.

2. T. Hattori: Sol. State Tech., 8 (July, 1990)

3. W. Zapka, W. Ziemlich, A.C. Tam: Appl. Phys. Lett. 58, 2217 (1991)

4. A.C. Tam, W.P. Leung, W. Zapka, W. Ziemlich: J. Appl. Phys. 71, 3515 (1992)

5. H.K. Park, C.P. Grigoropoulos, W.P. Leung, A.C. Tam: IEEE Trans. Comp., Pack., and Manuf. Techn. A 17, 631(1994)

6. Y.F. Lu, M. Takai, S. Komuro, T. Shiokawa, Y. Aoyagi: Appl. Phys. A, 59, 281 (1994)

7. K. Imen, S.J. Lee, S.D. Allen: Appl. Phys. Lett. 58, 203 (1991)

8. R. Oltra, O. Yavas, F. Cruz, J.P. Boquillon, C. Sartori: Appl. Surf. Sci. 96-98, 484 (1996)

9. J.A. Parrish, T.F. Deutsch: IEEE J. of Quant. Elect. 20, 12, 1386 (1984)

10. D. Bäuerle: Chemical Processing with Lasers (Springer, Berlin, 1996)

11. O. Yavas, R. Oltra, O. Kerrec: Appl. Phys. A,63, 321 (1996)

12. R. Oltra, G.M. Indrianjafy, J.P. Boquillon: J. de Phys. (Paris) IV C 7, 769 (1991).

13. R. Oltra, O. Yavas: Appl. Surf. Sci., (to appear)

14. P.T. Leung, N. Do, L. Klees, W.P. Leung, F. Tong, L. Lam, W. Zapka, A.C. Tam: J. Appl. Phys. 72, 2256 (1992)

15. O. Yavas, P. Leiderer, H.K. Park, C.P. Grigoropoulos, C.C. Poon, A.C. Tam: Phys. Rev. Lett. 70, 1830 (1993)

16. O. Yavas, P. Leiderer, H.K. Park, C.P. Grigoropoulos, C.C. Poon, A.C. Tam: Appl. Phys. A, 58, 407 (1994)

17. O. Yavas, P. Leiderer, H.K. Park, C.P. Grigoropoulos, C.C. Poon, A.C. Tam: Phys. Rev. Lett. 72, 2021 (1994)

18. O. Yavas: Laserinduzierte Gasblasennukleation (Hartung-Gorre, Konstanz, 1994)

19. J.C. Maxwell Garnett: Phil. Trans. Roy. Soc. London 203, 385 (1904)

20. D. Kim, H.K. Park, C.P. Grigoropoulos: Interferometric study on the growth of pulsed-laser-generated submicron bubble layer on a solid surface, National Heat Trans. Conf. Proc. Vol.4, pp.69-77, Houston, Texas, (1996)

21. H.K. Park, C.P. Grigoropoulos, C.C. Poon, A.C. Tam: Appl. Phys. Lett. 68, 596 (1996)

22. E. Kretschmann, H. Raether, Z. Naturf. A 23, 2135 (1968).

23. S. Herminghaus, P. Leiderer: Appl. Phys. A 51, 350 (1990)

24. H.K. Park, X. Xu, C.P. Grigoropoulos, N. Do, L. Klees, P.T. Leung, A.C. Tam: Appl. Phys. Lett. 61, 749 (1992)

25. P. Schiebener, J. Straub, J.M.H. Levelt Sengers, J.S. Gallagher: J. Phys. Chem. Ref. Data 19, 677 (1990)

26. P.B. Johnson, R.W. Christy: Phys. Rev. B 6, 4370 (1972) 
27. H.K. Park: Heat and Momentum Transfer on the Rapid Phase Change of Liquid Induced by Nanosecond-Pulsed Laser Irradiation (Dissertation, University of California at Berkeley, 1994)

28. S. Herminghaus, P. Leiderer: Appl. Phys. Lett. 58, 352 (1991)

29. S. Puderbach, S. Herminghaus, P. Leiderer: Phys. Lett. 130, 401(1988)

30. H.C.van de Hulst: Light Scattering by Small Particles (Dover, New York, 1981)

31. M. Born, E. Wolf: Principles of Optics (Pergamon, Oxford, 1991)
32. V.P. Carey: Liquid-Vapor Phase-Change Phenomena (Hemisphere, Washington, 1992)

33. A. Schilling, O. Yavas, J. Bischof, J. Boneberg, P. Leiderer: Appl. Phys. Lett. 69(27), 4159 (1996)

34. O. Yavas, A. Schilling, J. Bischof, J. Boneberg, P. Leiderer: Laser Phys., (submitted)

35. H.K. Park, D. Kim, C.P. Grigoropoulos, A.C. Tam: J. Appl. Phys. 80, 1 (1996) 